\title{
Laser Speckle Contrast Imaging to Evaluate Bowel Lesions in Neonates with NEC
}

\author{
Kristine Bach Korsholm Knudsen ${ }^{1,2}$ Joergen Thorup ${ }^{1,2}$ Rune Broni Strandby ${ }^{1,2}$ Rikard Ambrus $s^{1,2}$ \\ Linea Landgrebe Ring ${ }^{1,2}$ Inge Ifaoui ${ }^{1,2}$ \\ ${ }^{1}$ Department of Surgical Gastroenterology and Pediatric Surgery, \\ University Hospital of Copenhagen, Rigshospitalet, \\ Copenhagen, Denmark \\ 2 Faculty of Health and Medical Sciences, \\ University of Copenhagen, Denmark

\begin{abstract}
Address for correspondence Kristine Bach Korsholm Knudsen, Department of Surgical Gastroenterology and Pediatric Surgery, University Hospital of Copenhagen, Rigshospitalet, Blegdamsvej 9, Copenhagen 2100, Denmark (e-mail: kristine.bach.korsholm. knudsen@regionh.dk; inge.boetker.rasmussen.ifaoui@regionh.dk).
\end{abstract}

Eur J Pediatr Surg Rep 2017;5:e43-e46.

\begin{abstract}
Keywords

- necrotizing enterocolitis

- surgery

- infant

- microcirculation

- ischemia

Background Necrotizing enterocolitis (NEC) is the most frequent surgical emergency in newborns. Intestinal ischemia is considered a factor that precedes the development of NEC lesions. Laser speckle contrast imaging ( $(\mathrm{SCl})$ can be used to assess tissue microcirculation. We evaluated if $\mathrm{LSCl}$ may help to detect intestinal regions with reduced microcirculation in NEC.

Case Report A male patient (gestational age, 26 [3/7] weeks; birth weight, $600 \mathrm{~g}$ ) showed clinical signs of NEC 28 days after birth. X-ray revealed pneumatosis intestinalis and portal gas. Laparotomy showed NEC lesions with signs of transmural ischemia in the terminal ileum and cecum. Surgical resection lines (RLs) were marked, followed by $\mathrm{LSCl}$ measurements and resection of the bowel between the two RLs. Post hoc LSCI analyses were conducted on both sides of the proximal and distal RL. Low-flux values, indicating reduced microcirculation, were found in the macroscopically assessed necrotic bowel at the proximal RL, whereas higher flux values, indicating sufficient microcirculation, were found in the macroscopically assessed normal bowel.

Discussion This study is the first description of intra-abdominal use of $\mathrm{LSCl}$ to evaluate tissue microcirculation in relation to NEC lesions. $\mathrm{LSCl}$ could be a valuable tool to distinguish between ischemic and nonischemic bowel in neonates undergoing surgery for NEC.
\end{abstract}

\section{New Insights and the Importance for the Pediatric Surgeon}

This case report indicates that laser speckle contrast imaging (LSCI) may be a helpful tool to evaluate the extent of tissue ischemia in relation to surgery for NEC. LSCI may be used as an effective tool to better understand the etiology of NEC.

received

April 20, 2017

accepted after revision

July 16, 2017
DOI https://doi.org/

10.1055/s-0037-1606196.

ISSN 2194-7619. (c) 2017 Georg Thieme Verlag KG

Stuttgart · New York
License terms

(c) (1) 


\section{Introduction}

The exact etiology of NEC remains unclear, but intestinal ischemia may play an important role in the early pathogenesis. $^{1,2}$ However, it remains difficult to macroscopically detect tissue regions with ischemia, yet without discoloration, hemorrhage, or necrosis. Laser speckle contrast imaging (LSCI) was first introduced in the beginning of the 1980 s as a technique measuring noninvasive, real-time assessment of microcirculation. With this method, a speckle is generated after the surface of the tissue is illuminated with laser light. The movement of the blood cells induces fluctuations so that the microcirculation can be assessed as reported earlier. ${ }^{3-5}$ In this case report, intraoperative LSCI was tested in an infant with NEC to assess intestinal microcirculation in both the resected and remnant bowel regions.

\section{Case Report}

A preterm male was delivered by cesarean section at 26 weeks $(3 / 7)^{3 / 7}$ due to severe intrauterine growth restriction, preeclampsia, and reduced flow in the umbilical artery. The infant was transferred directly to the NICU. Birth weight was $600 \mathrm{~g}$, and Apgar scores were 9 and 10 at 1 and 10 minutes, respectively.

At birth, the infant underwent treatment with continuous positive pressure ventilation (CPAP) and a standardized regime with caffeine citrate, and intravenous fluid. During the second day of life, a pulmonic bleeding occurred with subsequent hypotension and respiratory failure. Intubation was indicated. On day 5, the infant improved clinically, and was returned to treatment with CPAP. On day 28 after birth, bloody stool and feeding intolerance were observed, and the infant was clinically described as irritated. Blood samples showed an elevated plasma lactate (3.6 $\mathrm{mmol} / \mathrm{L}$, normal range: $0.5-2.5 \mathrm{mmol} / \mathrm{L}$ ). Treatment with broadspectrum antibiotics was initiated and enteral feeding was stopped. Abdominal X-ray demonstrated portal gas with pneumatosis intestinalis (-Fig. 1). Despite the medical treatment, the child deteriorated further and became hemodynamically unstable. Therefore, a laparotomy was performed.

\section{Surgery}

The patient was placed in the supine position, and an exploratory laparotomy was performed. Intraoperative findings revealed intestinalis pneumatosis with subserosal gas bubbles, patchy NEC lesions involving the distal small bowel and ascending colon to the hepatic flexure, with macroscopic signs of transmural ischemia in the terminal ileum and cecum ( - Fig. 2). The surgeon marked the proximal and distal resection lines (RLs); then a LSCI exposure was taken as described below. The surgeon was blinded of the results. According to the procedure conducted in the department, all macroscopic nonviable intestine was resected; this included a right hemicolectomy and resection of $37 \mathrm{~cm}$ of the distal small intestine. An ileostomy and a mucous fistula were established.

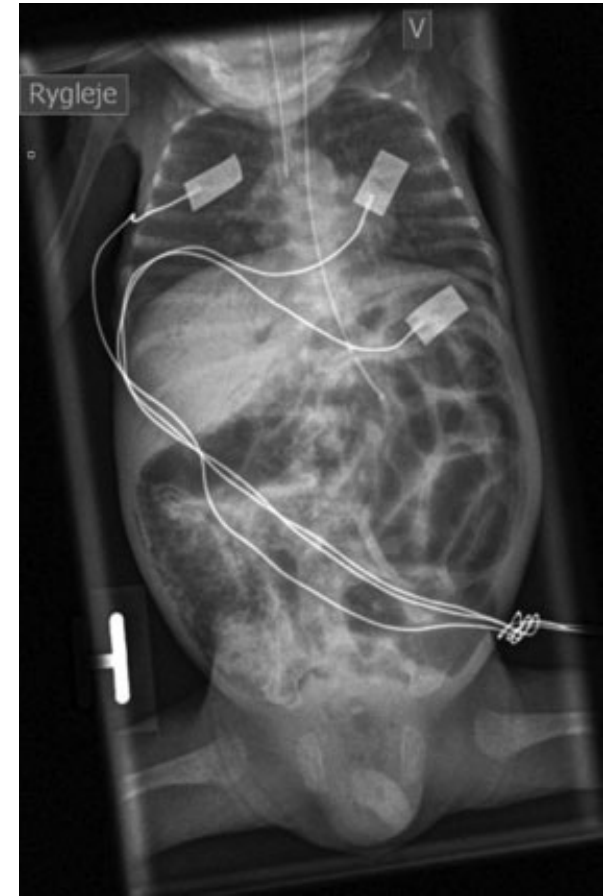

Fig. 1 Abdominal X-ray.

\section{Intraoperative $\mathbf{L S C I}$}

The LSCI instrument, which had a wavelength of $785 \mathrm{~nm}$ (MoorFLPI, Moor Instruments Ltd, Axminster, United Kingdom) was placed perpendicular to the tissue surface, $25 \mathrm{~cm}$ above the region of interest (ROI). Each ROI covered an area of $1 \mathrm{~cm}^{2}$. The recordings were made with high spatial resolution and low recording speed (sampling rate, $1 \mathrm{~Hz}$ ) to reduce variability. The obtained flux values were not used during surgery itself but were saved for later analysis. Post hoc LSCI analysis was performed each centimeter on both sides of the proximal RL

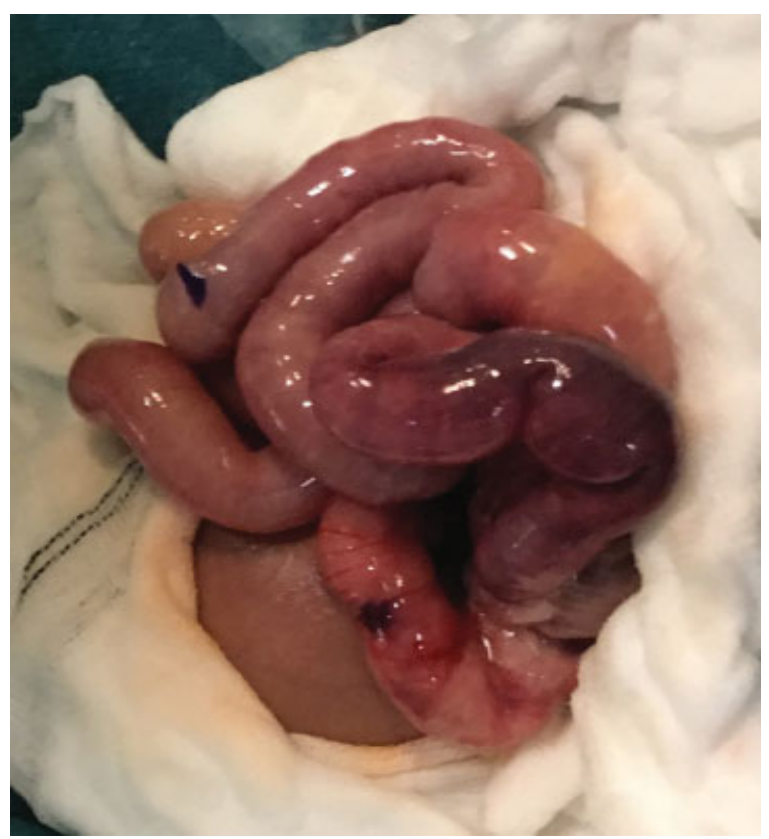

Fig. 2 Bowel with marked proximal and distal RL. RL, resection line. 


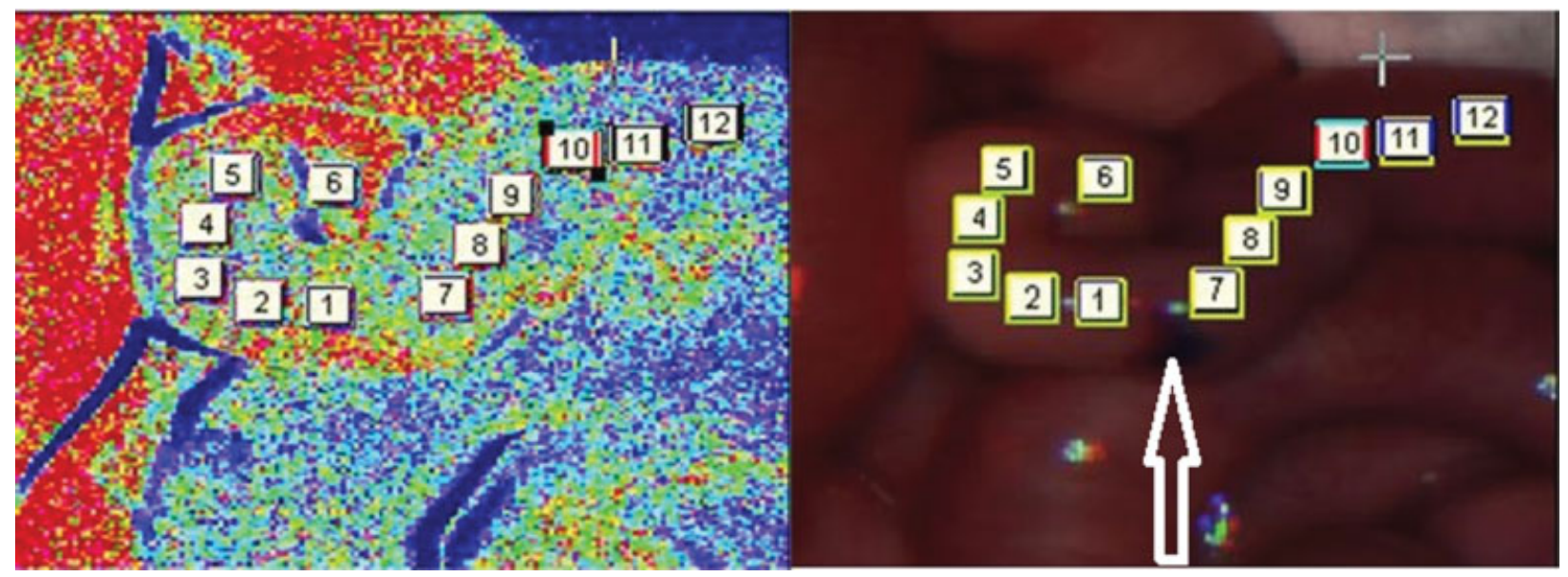

Fig. $3 \mathrm{LSCl}$ exposure and normal image of the proximal RL (marked with arrow). LSCl, laser speckle contrast imaging; RL, resection line.

from 1 to $6 \mathrm{~cm}$ at the proximal part relative to the distal part (-Fig. 3). At the anal RL, we measured on each side of the RL. Finally, an LSCl exposure was performed $15 \mathrm{~cm}$ proximal to the oral RL (macroscopically healthy bowel) and at the terminal ileum (necrotic bowel).

\section{Results}

The flux values are presented in -Fig. 4, and images of the bowel before and during LSCI exposure are presented in -Fig. 3. Consistent with apparent severe ischemia at the terminal ileum, where the lowest flux value was found at 155 laser speckle perfusion units (LSPU). Relatively low flux values were also measured at sites 3 to $4 \mathrm{~cm}$ proximally from the proximal RL and at sites 2 to $4 \mathrm{~cm}$ distally from the proximal RL, indicating that similar flux values were present on both sides of the proximal RL. Conversely, flux values were much higher $15 \mathrm{~cm}$ proximal to the proximal RL (macroscopic healthy bowel). Intermediate flux values were found on each side of the distal RL, proximal and distal parts, 585 and 450 LSPU, respectively. Histopathological examination of both the proximal and distal RL showed pneumatosis, necrotic mucosa, and preserved muscle layer.

The infant was clinically stable in the days after the operation. On postoperative day 14 (42 days after birth), the infant developed small bowel obstruction, and a second operation was performed. During laparotomy, a $10-\mathrm{cm}$ ischemic stenosis was identified in the small intestine, associated with the ileostomy, and a resection of the stenotic small bowel was performed.

The child recovered slowly, and after 8 weeks, an ileocolic anastomosis was performed.

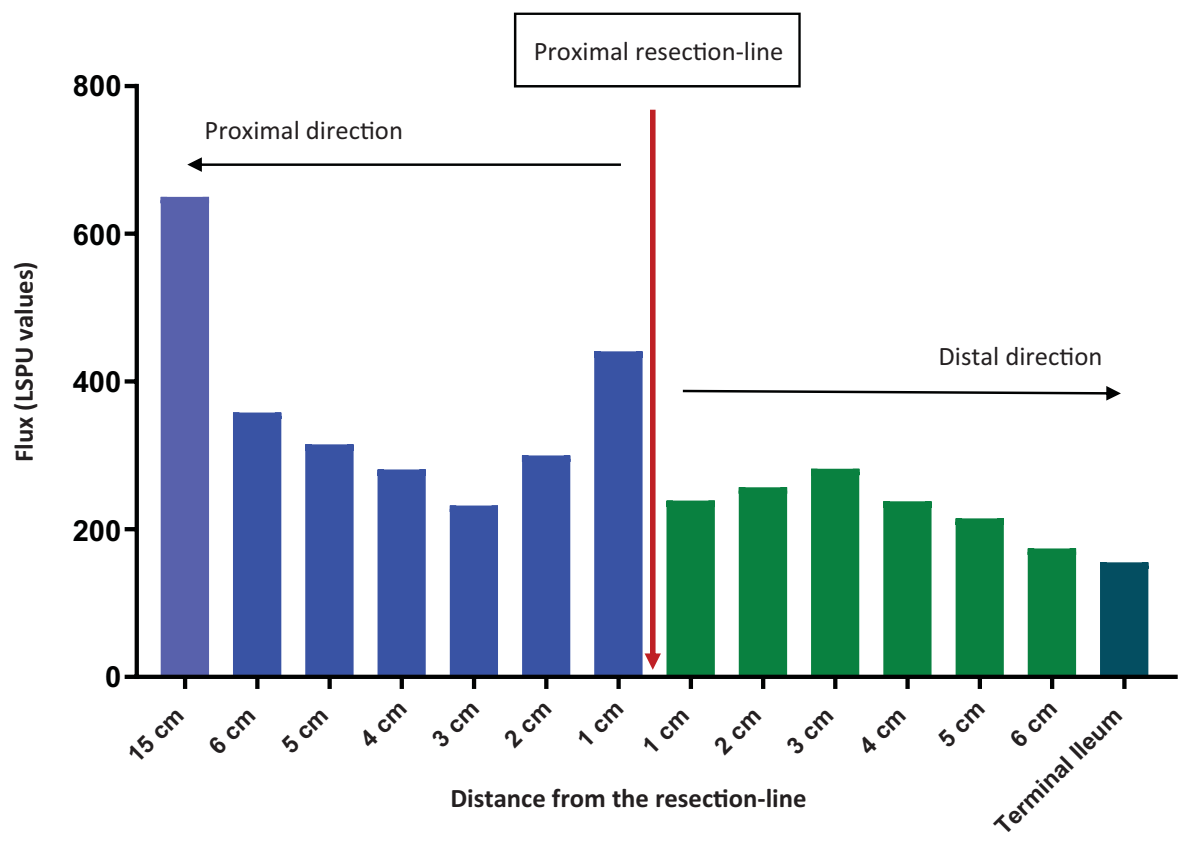

Fig. 4 Proximal RL with flux values. RL, resection line. 


\section{Discussion}

This case report indicates that LSCI may be a useful tool to evaluate the extent of tissue ischemia in relation to surgery for NEC. Decreased flux values, indicating decreased microcirculation, were demonstrated in bowel clearly affected by NEC, as judged by macroscopic inspection. These observations support earlier studies indicating that ischemia plays a role in the pathogenesis of NEC. ${ }^{1,6}$ We observed significant variations in flux values within a short section of the small intestine, adjacent to the necrotic sections. This indicates that ischemia may also play a role as an early trigger of NEC before macroscopic NEC lesions become evident. Low-flux values were found in the macroscopically assessed necrotic bowel compared with the higher flux values in macroscopically assessed normal bowel, though at the proximal RL, we found flux values in the intermittent area on both sides of the $\mathrm{RL}$ indicating reduced microcirculation at the proximal $\mathrm{RL}$, which was confirmed by the histological examination. This is interesting, since the surgeon found this part of the bowel to be viable based on the macroscopic findings. However, at the reoperation, 14 days after primary surgery, an ischemic stenosis was found in exactly this place. One might speculate that a reoperation could have been prevented if the results from the LSCI measurements had been used during surgery and more bowel resected. On the other hand, the LSCI measurements might also have been used to identify only irreversibly nonviable intestine in an effort to preserve as much bowel as possible together with a protective ileostomy. In this case, post hoc analyses were performed after operation; so, the surgeon was not aware of the results during surgery. This was done, since no "cutoff values" were known.

LSCI is a relatively easy and fast technique to use during laparotomy. Furthermore, it does not require tissue contact, like other techniques, such as Doppler flowmetry or indocyanine green, to measure perfusion. ${ }^{7}$

It is often difficult macroscopically to distinguish between viable and necrotic tissue, and LSCI may be a useful diagnostic tool in this context. Further observations are clearly needed to define a relevant cutoff value for flux values in different bowel regions. The variation in flux values within a short section of the small intestine may indicate that the ischemia in connection with NEC is not mainly caused by generalized hypoxia but rather by local factors contributing to ischemia. Local inflammation has been proposed as an important factor for NEC development, as it can induce ischemia via mediators, such as platelet activation factor, toll-like receptor 4, and leukocyte infiltration. ${ }^{8,9}$ As such, LSCI may be used as an effective tool to better understand the etiology of NEC as well as help clinical diagnosis during NEC surgery. Further, well-controlled LSCI studies in an animal model of NEC, such as the preterm pig, ${ }^{10}$ which allows surgical interventions during NEC, coupled with further LSCI studies in infants, may help to establish a new tool to support NEC prevention and recovery.

\section{Conflict of Interest}

None.

\section{References}

1 Ballance WA, Dahms BB, Shenker N, Kliegman RM. Pathology of neonatal necrotizing enterocolitis: a ten-year experience. J Pediatr 1990;117(1, Pt 2):S6-S13

2 Högberg N, Carlsson PO, Hillered L, Stenbäck A, Lilja HE. Intraluminal intestinal microdialysis detects markers of hypoxia and cell damage in experimental necrotizing enterocolitis. J Pediatr Surg 2012;47(09):1646-1651

3 Ambrus R, Svendsen LB, Secher NH, et al. A reduced gastric corpus microvascular blood flow during Ivor-Lewis esophagectomy detected by laser speckle contrast imaging technique. Scand J Gastroenterol 2017;52(04):455-461

4 Richards LM, Towle EL, Fox DJ Jr, Dunn AK. Intraoperative laser speckle contrast imaging with retrospective motion correction for quantitative assessment of cerebral blood flow. Neurophotonics 2014;1(01):015006

5 Ponticorvo A, Cardenas D, Dunn AK, Ts'o D, Duong TQ. Laser speckle contrast imaging of blood flow in rat retinas using an endoscope. J Biomed Opt 2013;18(09):090501

6 Schat TE, Heida FH, Schurink M, et al. The relation between splanchnic ischaemia and intestinal damage in necrotising enterocolitis. Arch Dis Child Fetal Neonatal Ed 2016;pii: fetalneonatal-2015-309838

7 Ambrus R, Strandby RB, Svendsen LB, Achiam MP, Steffensen JF, Søndergaard Svendsen MB. Laser speckle contrast imaging for monitoring changes in microvascular blood flow. Eur Surg Res 2016;56(3-4):87-96

8 Chen Y, Chang KT, Lian DW, et al. The role of ischemia in necrotizing enterocolitis. J Pediatr Surg 2016;51(08):1255-1261

9 Federici S, DE Biagi L, Straziuso S, et al. Multicenter retrospective study on management and outcome of newborns affected by surgical necrotizing enterocolitis. Minerva Chir 2017;72(03): 183-187

10 Sangild PT, Thymann T, Schmidt M, Stoll B, Burrin DG, Buddington RK. Invited review: the preterm pig as a model in pediatric gastroenterology. J Anim Sci 2013;91(10):4713-4729 